PROCEEDINGS OF THE

AMERICAN MATHEMATICAL SOCIETY

Volume 135, Number 2, February 2007, Pages 551-558

S 0002-9939(06)08477-2

Article electronically published on August 1, 2006

\title{
CARRIER AND NERVE THEOREMS IN THE EXTENSION THEORY
}

\author{
ANDRZEJ NAGÓRKO \\ (Communicated by Alexander Dranishnikov)
}

\begin{abstract}
We show that a regular cover of a general topological space provides structure similar to a triangulation. In this general setting we define analogues of simplicial maps and prove their existence and uniqueness up to homotopy. As an application we give simple proofs of sharpened versions of nerve theorems of K. Borsuk and A. Weil, which state that the nerve of a regular cover is homotopy equivalent to the underlying space.

Next we prove a nerve theorem for a class of spaces with uniformly bounded extension dimension. In particular we prove that the canonical map from a separable metric $n$-dimensional space into the nerve of its weakly regular open cover induces isomorphisms on homotopy groups of dimensions less than $n$.
\end{abstract}

\section{INTRODUCTION}

An efficient way to investigate properties of a topological space is to divide it into pieces and examine how they are glued together. We show how to divide a general topological space and endow it with a structure that resembles a triangulation. We employ this analogy to sharpen prior results of homotopy theory, known as carrier and nerve theorems.

In order to divide a space that belongs to a class $\mathcal{C}$ of topological spaces we must decide what a piece is. We want it to resemble a simplex as much as possible. As a simplex is an archetype of an absolute extensor, the choice of absolute extensors for $\mathcal{C}$ as pieces is quite natural.

Definition. A space $Y$ is an absolute extensor for a space $X$ if each map from a closed subset of $X$ into $Y$ extends over the entire space $X$. The class of absolute extensors for all spaces from a class $\mathcal{C}$ is denoted by $A E(\mathcal{C})$. We write $A E(X)$ to abbreviate $A E(\{X\})$.

The following defines regular covers that endow a space with structures similar to triangulations. Recall that a cover is locally finite dimensional if its nerve is such.

Definition. Let $\mathcal{C}$ be a class of topological spaces. We say that a cover is a $\mathcal{C}$-cover if the intersection of each non-empty collection of its elements belongs to $\mathcal{C}$. A

Received by the editors May 16, 2005 and, in revised form, August 19, 2005.

2000 Mathematics Subject Classification. Primary 54C20; Secondary 54F45, 55P10.

Key words and phrases. Carrier theorem, nerve theorem, regular cover, absolute extensor.

The author is grateful to Professor Henryk Toruńczyk for his advice while preparing this paper.

(C)2006 American Mathematical Society

Reverts to public domain 28 years from publication 
locally finite $A E(\mathcal{C})$-cover that is either closed and locally finite dimensional or is open is said to be regular for the class $\mathcal{C}$.

Examples of regular covers include a locally finite cover of a Euclidean space by its open balls and a cover of a finite simplicial complex by its simplices.

The main result of this paper states that the nerve of a regular cover reflects the homotopy structure of the underlying space. The result is extended to classes of spaces with bounded extension dimension, where appropriate theory of $[L]$ homotopy equivalences is used. Apart from generalizations, the unified approach developed in this paper delivers simplifications of proofs of earlier known theorems. In particular our carrier theorems imply nerve theorems of K. Borsuk [3, p. 234] and A. Weil [15, p. 141], while Theorem 3.4 generalizes an $n$-connectivity nerve theorem recently proved by A. Björner [2]

More specialized definitions of covers that by our definition are regular were already used in the literature. K. Kawamura gave characterizations of infinite dimensional manifolds in terms of partitions [11. G. de Rham used covers by convex subsets to define simple homotopy types of Riemannian manifolds [5. Finally nerve theorems are often used as a bridge between combinatorics and topology [1. Our generalization is motivated by the application of regular covers in the recent proof of characterization and rigidity theorems for Nöbeling manifolds [14. In 4] A. Chigogidze conjectured that analogous characterizations hold for universal spaces for extension dimension. At the end of section 3 we give a nerve theorem for spaces of bounded extension dimension as a first step in a program to prove these conjectures.

\section{CARRIER THEOREMS}

How to extend a partial map from a subcomplex to the entire CW complex? If each map from the boundary of a Euclidean ball into the codomain extends over the ball, then the answer is easy: order cells by inclusion and construct an extension inductively. But the asphericity of the codomain (vanishing of all its homotopy groups) is a rare luxury. The same technique would work though if we were able to restrict ranges of the map on individual cells of the CW complex to aspherical subspaces of the codomain. This idea leads to the notion of a carrier and to the aspherical carrier theorem [12, II §9]. We generalize this notion to arbitrary spaces using a cover to replace the cell structure in the domain.

Definition. A carrier is a function $C: \mathcal{F} \rightarrow \mathcal{G}$ from a cover $\mathcal{F}$ of a space $X$ into a collection $\mathcal{G}$ of subsets of a topological space such that for each $\mathcal{A} \subset \mathcal{F}$ if $\bigcap \mathcal{A} \neq \emptyset$, then $\bigcap_{A \in \mathcal{A}} C(A) \neq \emptyset$. We say that a map $f$ is carried by $C$ if it is defined on a closed subset of $X$ and $f(F) \subset C(F)$ for each $F \in \mathcal{F}$ (we write $f(F)$ for $f(F \cap \operatorname{dom} f)$ ).

Carrier Theorem. Assume that $C: \mathcal{F} \rightarrow \mathcal{G}$ is a carrier such that $\mathcal{F}$ is a closed cover of a space $X$ and $\mathcal{G}$ is an $A E(X)$-cover of another space. If $\mathcal{F}$ is locally finite and locally finitely dimensional, then each map carried by $C$ extends to a map of the entire space $X$, also carried by $C$.

Special cases of the Carrier Theorem follow from Michael's Selection Theorem, as the multivalued map given by the formula $F(x)=\bigcap_{F \ni x} C(F)$ is lower semicontinuous.

The proof of the Weak Carrier Theorem (later in this section) is similar to the proof of the Carrier Theorem and can be read simultaneously. The key difference 
is in the definition and an order of sets $\delta_{\gamma}$. The reader may compare both cases for a cover $\mathcal{F}$ that consists of three sets with non-empty intersection.

Proof. Let $f_{0}$ be a map carried by $C$ and let $A=\operatorname{dom} f_{0}$. Let $\left\{\mathcal{E}_{\gamma}\right\}_{0<\gamma<\Gamma}$ be a transfinite sequence of all subcollections of $\mathcal{F}$ with non-empty intersections, such that the sequence $\left\{\delta_{\gamma}=\bigcap \mathcal{E}_{\gamma}\right\}_{0<\gamma<\Gamma}$ is non-decreasing in the order by inclusion. Its existence is guaranteed by the assumption of local finite dimensionality of $\mathcal{F}$. Let $C_{\gamma}=\bigcap_{E \in \mathcal{E}_{\gamma}} C(E)$ and observe that an arbitrary map $f$ is carried by $C$ if and only if $f\left(\delta_{\gamma}\right) \subset C_{\gamma}$ for each $0<\gamma<\Gamma$. Let $\delta_{\Gamma}=\emptyset$. We shall construct a transfinite sequence of maps $\left\{f_{\gamma}: A \cup \bigcup_{0<\alpha<\gamma} \delta_{\alpha} \rightarrow Y\right\}_{\gamma \leq \Gamma}$ such that $f_{\alpha}$ extends $f_{\beta}$ for all $0 \leq \beta \leq \alpha \leq \Gamma$ and $f_{\gamma}\left(\delta_{\gamma}\right) \subset C_{\gamma}$ for each $0<\gamma<\Gamma$. The map $f_{\Gamma}$ will be an extension that we are looking for, since $\bigcup_{\gamma<\Gamma} \delta_{\gamma}=X$.

We proceed by transfinite induction. Fix $\gamma \leq \Gamma$ and assume that for each $\alpha<\gamma$ we already constructed $f_{\alpha}$. The map $f_{\gamma}^{\prime}=\bigcup_{\alpha<\gamma} f_{\alpha}$ is well defined, continuous and its domain is closed in $X$ because maps $f_{\alpha}$ agree on intersections of their domains and $\mathcal{F}$ is closed and locally finite. If $\gamma=\Gamma$, then $\delta_{\gamma}=\emptyset$ and we may put $f_{\Gamma}=f_{\Gamma}^{\prime}$. If $\gamma<\Gamma$, then by the order of $\delta_{\gamma}$ and by inductive assumptions $f_{\gamma}^{\prime}$ maps $\delta_{\gamma}$ into $C_{\gamma}$. The set $C_{\gamma}$ is non-empty because $C$ is a carrier and is an absolute extensor for $\delta_{\gamma}$ because $\mathcal{G}$ is an $A E(X)$-cover. So $f_{\gamma}^{\prime}$ extends onto $\delta_{\gamma}$ to a map $f_{\gamma}$ such that $f_{\gamma}\left(\delta_{\gamma}\right) \subset C_{\gamma}$ and our construction is finished.

Definition. A cover is regular for a space $X$ if it is regular for the class $\{X\}$.

Corollary 2.1. If a closed cover $\mathcal{G}$ of a space $Y$ is regular for $X \times[0,1]$, then every two $\mathcal{G}$-close maps from $X$ into $Y$ are $\mathcal{G}$-homotopic. Moreover such homotopy exists with an additional property that if endpoints of its path lie in an element of $\mathcal{G}$, then the entire path lies in it.

Proof. Let $f$ and $g$ denote $\mathcal{G}$-close maps from $X$ into $Y$. Let $\mathcal{F}=\left\{F_{G}\right\}_{G \in \mathcal{G}}$ be the collection of subsets of $X \times[0,1]$ defined by

$$
F_{G}=\left(f^{-1}(G) \cap g^{-1}(G)\right) \times[0,1] .
$$

It is a cover of $X \times[0,1]$ because $f$ and $g$ are $\mathcal{G}$-close. Define a carrier $C: \mathcal{F} \rightarrow \mathcal{G}$ by the formula $C\left(F_{G}\right)=G$ and a map $F: X \times\{0,1\} \rightarrow Y$ by $F(x, 0)=f(x)$ and $F(x, 1)=g(x)$. By definition, $F$ is carried by $C$ and by the Carrier Theorem it admits an extension to the entire space $X \times[0,1]$, also carried by $C$. This extension is a $\mathcal{G}$-homotopy that satisfies our claim.

In applications (see [11, 2]), covers with slightly weaker regularity conditions are sometimes used, as in the following definition.

Definition. Let $\mathcal{C}$ be a class of topological spaces. We say that a cover is a weak $\mathcal{C}$ cover if the union of each collection of its elements that has a non-empty intersection belongs to $\mathcal{C}$. A locally finite weak $A E(\mathcal{C})$-cover that is either closed and locally finite dimensional or is open is said to be weakly regular for the class $\mathcal{C}$.

It follows from the Carrier Theorem that a regular cover is a weakly regular cover, but the converse is not true.

Definition. Let $\mathcal{F}$ be a cover of a space $X$ and let $C: \mathcal{F} \rightarrow \mathcal{G}$ be a carrier. We say that a map $f$ is weakly carried by $C$ if it is defined on a closed subset of $X$ and for each $x \in \operatorname{dom} f$ there exists an $F \in \mathcal{F}$ such that $x \in F$ and $f(x) \in C(F)$. 
If $\mathcal{F}$ is a cover of a space $X$ and $i d_{\mathcal{F}}$ is the identity map of $\mathcal{F}$, then a map from $X$ into $X$ is weakly carried by $i d_{\mathcal{F}}$ if and only if it is $\mathcal{F}$-close to the identity of $X$. By this observation the composition of two maps weakly carried by $i d_{\mathcal{F}}$ does not have to be weakly carried by it.

Weak Carrier Theorem. Assume that $C: \mathcal{F} \rightarrow \mathcal{G}$ is a carrier such that $\mathcal{F}$ is an open cover of a space $X$ and $\mathcal{G}$ is a weak $A E(X)$-cover of another space. If $\mathcal{F}$ is locally finite, then each map weakly carried by $C$ extends to a map of the entire space $X$, also weakly carried by $C$.

Proof. Let $f_{0}$ be a map weakly carried by $C$ and let $A=\operatorname{dom} f_{0}$. Let $\left\{\mathcal{E}_{\gamma}\right\}_{0<\gamma<\Gamma}$ be a transfinite sequence of all subcollections of elements of $\mathcal{F}$ with non-empty intersections, non-decreasing in the order by inclusion. For each $0<\gamma<\Gamma$ let $\delta_{\gamma}$ be the set of points in $X$ that belong exactly to all elements of $\mathcal{E}_{\gamma}$, that is, $\delta_{\gamma}=\bigcap \mathcal{E}_{\gamma} \backslash \bigcup\left(\mathcal{F} \backslash \mathcal{E}_{\gamma}\right)$. Let $C_{\gamma}=\bigcup_{E \in \mathcal{E}_{\gamma}} C(E)$ and observe that an arbitrary map $f$ is weakly carried by $C$ if and only if $f\left(\delta_{\gamma}\right) \subset C_{\gamma}$ for each $0<\gamma<\Gamma$. Let $\delta_{\Gamma}=\emptyset$. We shall construct a transfinite sequence of maps $\left\{f_{\gamma}: A \cup \cup_{0<\alpha \leq \gamma} \delta_{\alpha} \rightarrow Y\right\}_{\gamma \leq \Gamma}$ such that $f_{\alpha}$ extends $f_{\beta}$ for all $\beta \leq \alpha \leq \Gamma$ and $f_{\gamma}\left(\delta_{\gamma}\right) \subset C_{\gamma}$ for each $0<\gamma<\Gamma$. The map $f_{\Gamma}$ will be an extension that we are looking for, since $\bigcup_{\gamma<\Gamma} \delta_{\gamma}=X$.

We proceed by transfinite induction. Fix $\gamma \leq \Gamma$ and assume that for each $\alpha<\gamma$ we already constructed $f_{\alpha}$. The map $f_{\gamma}^{\prime}=\bigcup_{\alpha<\gamma} f_{\alpha}$ is well defined and continuous because maps $f_{\alpha}$ agree on intersections of their domains and $\mathcal{F}$ is locally finite. By the definition of the order of sets $\delta_{\gamma}$ its domain is closed. If $\gamma=\Gamma$, then $\delta_{\gamma}=\emptyset$ and we may put $f_{\Gamma}=f_{\Gamma}^{\prime}$. If $\gamma<\Gamma$, then by the order of $\delta_{\gamma}$ and inductive assumptions $f_{\gamma}^{\prime}$ maps $\delta_{\gamma}$ into $C_{\gamma}$. The set $C_{\gamma}$ is an absolute extensor for $\delta_{\gamma}$ because it is a union of elements of $\mathcal{G}$ that have non-empty intersection and $\mathcal{G}$ is a weak $A E(X)$-cover. So $f_{\gamma}^{\prime}$ extends onto $\delta_{\gamma}$ to a map $f_{\gamma}$ such that $f_{\gamma}\left(\delta_{\gamma}\right) \subset C_{\gamma}$ and our construction is finished.

Corollary 2.2. If an open cover $\mathcal{G}$ of a space $Y$ is weakly regular for $X \times[0,1]$, then every two $\mathcal{G}$-close maps from $X$ into $Y$ are st $\mathcal{G}$-homotopic, where st $\mathcal{G}$ denotes the star of $\mathcal{G}$. Moreover such homotopy exists with an additional property that if endpoints of its path lie in an element of $\mathcal{G}$, then the entire path lies in its star.

We omit the proof as it is similar to the proof of Corollary 2.1

Remark. In the Weak Carrier Theorem the assumption that $\mathcal{F}$ is locally finite may be omitted if $\mathcal{G}$ is open and $X$ is paracompact. To prove this, it suffices to find a locally finite open refinement $\mathcal{H}$ of $\mathcal{F}$ and a carrier $D: \mathcal{H} \rightarrow \mathcal{F}$ such that $f$ is weakly carried by $C \circ D$ and $H \subset D(H)$ for each $H \in \mathcal{H}$. Then, by the Weak Carrier Theorem, $f$ extends over $X$ to a map weakly carried by $C \circ D$, which is obviously weakly carried by $C$. Let $\mathcal{H}$ be any locally finite refinement of an open cover $\left\{F \cap f^{-1}(C(F)): F \in \mathcal{F}\right\}$ and let $D: \mathcal{H} \rightarrow \mathcal{F}$ be any map such that $H \subset D(H) \cap f^{-1}(C(D(H)))$ for each $H \in \mathcal{H}$. Then $f$ is carried by $C \circ D$ because for each $x \in X$ there exists $H \in \mathcal{H}$ such that $x \in H \in D(H) \cap f^{-1}(C(D(H)))$ so $f(x) \in(C \circ D)(H)$. By the definition $f$ is weakly carried by $C \circ D$ and the proof is finished.

Similarly in Corollary 2.2 the assumption of local finiteness of $\mathcal{G}$ may be replaced by paracompactness of $X$. 


\section{NERVE THEOREMS}

Nerve theorems give conditions under which the nerve of a cover is equivalent to the underlying space. First examples of such theorems are attributed to K. Borsuk [3, p. 234] (for closed covers) and A. Weil [15, p. 141] (for open covers), both for homotopy equivalences. Since then, several generalizations were made. First generalizations by W. Holsztyński [9] and J. N. Haimov [8] relaxed conditions on the cover. Next weak homotopy equivalences were studied in this context by M. McCord [13] and weak $n$-homotopy equivalences by A. Björner [2].

Let $K$ denote an arbitrary simplicial complex. An open star st $v$ of a vertex $v \in$ $K$ is the complement of the union of all simplices of $K$ that do not contain $v$. A barycentric star bst $v$ of a vertex $v \in K$ is the union of all simplices of the first barycentric subdivision of $K$ that contain $v$.

Lemma 3.1. If the cover of a simplicial complex, with the metric topology, by the collection of open (or barycentric) stars of its vertices is locally finite (and locally finite dimensional), then it is regular for the class of metric spaces. If additionally the complex is locally countable, then the cover is regular for the class of normal spaces.

We omit details of the proof, which is based on 10, Theorem 11.7, p. 108, and Theorem 7.1, p. 43] and the observation that the intersection of a collection of stars is star-shaped. Every point of the intersection is connected by a line with the barycenter of centers of stars that are intersected.

We shall use the following notation.

Definition. Let $\mathcal{S}_{\mathcal{F}}$ denote the collection of open stars of vertices of the nerve $N(\mathcal{F})$ of a point-finite cover $\mathcal{F}$. Let $\mathcal{B}_{\mathcal{F}}$ denote the collection of its barycentric stars.

By Lemma 3.1. covers $\mathcal{S}_{\mathcal{F}}$ and $\mathcal{B}_{\mathcal{F}}$ are regular for the class of normal spaces. Their structure mirrors the structure of $\mathcal{F}$, in the sense of the following definition.

Definition. We say that covers are isomorphic if there exists a carrier from one of them onto the other, which is invertible (i.e., is bijective and its inverse is a carrier).

Observe that if the codomain of a carrier $C$ is equal to the domain of a carrier $D$, then a composition of two maps carried respectively by $C$ and $D$ is carried by $D \circ C$.

Lemma 3.2. Let $\mathcal{F}$ be a point-finite cover and let $v(F)$ denote the vertex of $N(\mathcal{F})$ corresponding to a set $F \in \mathcal{F}$. The functions $S: \mathcal{F} \rightarrow \mathcal{S}_{\mathcal{F}}$ and $B: \mathcal{F} \rightarrow \mathcal{B}_{\mathcal{F}}$ defined by $S(F)=\operatorname{st} v(F)$ and $B(F)=$ bst $v(F)$ are invertible carriers.

Proof. Obviously $S$ and $B$ are bijections. We shall prove that $B$ and $S^{-1}$ are carriers. Then, since a function $I: \mathcal{B}_{\mathcal{F}} \rightarrow \mathcal{S}_{\mathcal{F}}$ defined by the formula $I$ (bst $\left.v\right)=$ st $v$ is a carrier and $S^{-1} \circ I \circ B=i d_{\mathcal{F}}$, functions $B^{-1}=S^{-1} \circ I$ and $S=I \circ B$ are carriers too.

To prove that $B$ is a carrier, assume that $\left\{F_{i}\right\}$ is a collection of elements of $\mathcal{F}$ with non-empty intersection. Then the nerve $N(\mathcal{F})$ contains a simplex $\sigma$ spanned by vertices $\left\{v\left(F_{i}\right)\right\}$ and each barycentric star bst $v\left(F_{i}\right)$ contains the barycenter of $\sigma$. Therefore the intersection of $\left\{B\left(F_{i}\right)\right\}$ is non-empty.

To prove that $S^{-1}$ is a carrier, assume that $\left\{\right.$ st $\left.v\left(F_{i}\right)\right\}$ is a collection of elements of $\mathcal{S}_{\mathcal{F}}$ with non-empty intersection. As an open star of a vertex $v\left(F_{i}\right)$ contains only points of simplices that contain $v\left(F_{i}\right)$, then the intersection of the set $\left\{\operatorname{st} v\left(F_{i}\right)\right\}$ 
contains only points of simplices that contain all vertices $v\left(F_{i}\right)$. So the nerve $N(\mathcal{F})$ contains a simplex spanned by $\left\{v\left(F_{i}\right)\right\}$ and the intersection of $\left\{F_{i}\right\}$ is non-empty.

Putting everything together we obtain nerve theorems for homotopy equivalences. We state the theorem for closed covers, which generalizes a nerve theorem by J. N. Haimov 8. An analogous theorem for open covers may also be proved. We do not state it here, as it turns out to be equivalent to the nerve theorem by A. Weil [15.

Theorem 3.3. Assume that a closed cover $\mathcal{F}$ of a normal space $X$ is regular for the class of metric spaces. If $\mathcal{F}$ is star-countable, then $X$ and the nerve of $\mathcal{F}$ are homotopy equivalent.

The main theorem of [8] states the same conclusion under the additional assumption that $\mathcal{F}$ is star-finite and $X$ is paracompact.

Proof. Let $B: \mathcal{F} \rightarrow \mathcal{B}_{\mathcal{F}}$ be an invertible carrier as defined in Lemma 3.2, By the Carrier Theorem and Lemma 3.1 there exist $f: X \rightarrow N(\mathcal{F})$ and $g: N(\mathcal{F}) \rightarrow X$ carried by $B$ and $B^{-1}$ respectively. Then $g \circ f$ is carried by $B^{-1} \circ B$ so it is $\mathcal{F}$-close to $i d_{X}$ and by Corollary $2.1 \mathrm{~g}$ is a homotopy inverse of $f$. Analogously $f$ is a homotopy inverse of $g$ so $X$ and $N(\mathcal{F})$ are homotopy equivalent.

We turn our attention to spaces with bounded dimension. First we prove a nerve theorem for the class of at most $n$-dimensional spaces. Next the case of extension dimension is studied. To avoid anomalies of the dimension of general topological spaces from now on all spaces are assumed to be separable metric.

Theorem 3.4. If $\mathcal{F}$ is an open cover of a space $X$, weakly regular for the class of at most $n$-dimensional spaces, then each canonical map $\varkappa: X \rightarrow N(\mathcal{F})$ induces isomorphisms on homotopy groups of dimensions less than $n$.

A canonical map into the nerve of a cover is a map induced by a partition of unity subordinated to this cover, by interpreting its values as barycentric coordinates of points in the nerve. Kuratowski's $\varkappa$-map [7, p. 321] is an example of such a map.

It follows from the Excision Theorem that an open cover $\mathcal{F}$ of a locally $(n-1)$ connected space is weakly regular for the class of at most $n$-dimensional spaces if and only if an intersection of each collection $\mathcal{A} \subset \mathcal{F}$ is $(n-|\mathcal{A}|)$-connected. This relates our theorem to Björner's nerve theorem [2].

Proof. For each subcollection $\mathcal{A}$ of $\mathcal{F}$ with non-empty intersection let $v(\mathcal{A})$ denote the simplex of the nerve $N(\mathcal{F})$ spanned by vertices $\{v(A)\}_{A \in \mathcal{A}}$.

Let $\lambda: N(\mathcal{F})^{(n)} \rightarrow X$ denote a map from the $n$-dimensional skeleton of $N(\mathcal{F})$ into $X$, weakly carried by the carrier $S^{-1}$ defined in Lemma 3.2 Fix $k<n$. For each map $\varphi: S^{k} \rightarrow N(\mathcal{F})$ pick a map $\varphi^{\prime}: S^{k} \rightarrow N(\mathcal{F})^{(n)}$, homotopic to $\varphi$, such that if $\varphi(x) \in \sigma$, then $\varphi^{\prime}(x) \in \sigma^{(n)}$ for each simplex $\sigma$ of $N(\mathcal{F})$. It exists by the Cellular Approximation Theorem.

To prove that $\varkappa$ induces an epimorphism on $k$ th homotopy groups we shall observe that for each $\varphi: S^{k} \rightarrow N(\mathcal{F})$ the map $\varkappa \circ\left(\lambda \circ \varphi^{\prime}\right)$ is $\mathcal{S}_{\mathcal{F}}$-close to $\varphi$, so by Corollary 2.2 they are homotopic. Fix $x \in S^{k}$. From the definition of a weakly carried map there exists $F \in \mathcal{F}$ such that $\varphi^{\prime}(x) \in$ st $v(F)$ and $\lambda\left(\varphi^{\prime}(x)\right) \in F$. From the definition of $\varphi^{\prime}$ we have $\varphi(x) \in$ st $v(F)$. From the definition of $\varkappa$ we have $\varkappa(F) \subset \operatorname{st} v(F)$. Then $\varkappa\left(\lambda\left(\varphi^{\prime}(x)\right)\right) \in$ st $v(F)$ and we are done. 
To prove that $\varkappa$ induces a monomorphism on $k$ th homotopy groups we shall observe that for each $\psi: S^{k} \rightarrow X$ the map $\lambda \circ\left((\varkappa \circ \psi)^{\prime}\right)$ is $\mathcal{F}$-close to $\psi$, so by Corollary 2.2 they are homotopic, as dimension of $S^{k} \times[0,1]$ is at most $n$. Fix $x \in S^{k}$ and let $\mathcal{A}=\{F \in \mathcal{F}: \psi(x) \in F\}$. Then $(\varkappa \circ \psi)(x) \in v(\mathcal{A})$ so $(\varkappa \circ \psi)^{\prime}(x) \in v(\mathcal{A})^{(n)}$. By the definition of $\mathcal{S}_{\mathcal{F}}, v(\mathcal{A}) \cap \operatorname{st} v(F) \neq \emptyset$ if and only if $F \in \mathcal{A}$, so $\lambda \circ(\varkappa \circ \psi)^{\prime}(x) \in F$ for some $F \in \mathcal{A}$. Therefore if $\varkappa \circ \psi$ is null-homotopic, then $\lambda \circ\left((\varkappa \circ \psi)^{\prime}\right)$ and so is $\psi$.

Remark. Let $\sigma(\mathcal{A})=\operatorname{Cl}(\bigcap \mathcal{A}) \backslash \bigcup(\mathcal{F} \backslash \mathcal{A})$ be the closure of the set of points in $X$ that belong exactly to elements of $\mathcal{A}$. Then the map $K(\sigma(\mathcal{A}))=v(\mathcal{A})$, where $\mathcal{A}$ runs over all subcollections of $\mathcal{F}$ that have non-empty intersections, is a carrier. To prove that, observe that if $x \in \bigcap_{i} \sigma\left(\mathcal{A}_{i}\right)$, then $\mathcal{A}=\{F \in \mathcal{F}: x \in F\} \subset \mathcal{A}_{i}$ for each $i$, so $\bigcap_{i} v\left(\mathcal{A}_{i}\right) \supset v(\mathcal{A}) \neq \emptyset$. Every canonical map into the nerve of a cover is carried by the carrier $K$.

We finish with a generalization of Theorem 3.4 to a class of spaces with uniformly bounded extension dimension. The survey [4 is a good source of information about notions of the extension dimension and the $[L]$-homotopy; here we will only recall basic definitions.

Definition. Let $L$ be an arbitrary CW complex. A space $X$ has extension dimension less than or equal to $[L]$ if $L$ is an absolute extensor for $X$. The class of absolute extensors for at most $[L]$-dimensional spaces is denoted by $A E[L]$.

We say that maps $f, g: X \rightarrow Y$ are $[L]$-homotopic if for each at most $[L]$ dimensional space $Z$, each pair $A, B$ of disjoint closed subsets of $Z$ and each map $h: Z \rightarrow X$ there exists an extension of a map $f \circ h_{\mid A} \cup g \circ h_{\mid B}$ to the entire space $Z$.

We shall need the following analogue of Corollary 2.2

Lemma 3.5. If $\mathcal{G}$ is a locally finite closed weak $A E[L]$-cover of a separable metric space, then every two $\mathcal{G}$-close maps are $[L]$-homotopic.

Proof. Name the maps considered by $f, g: X \rightarrow Y$, where $Y$ denotes the space covered by $\mathcal{G}$. We are going to prove that $f$ and $g$ are $[L]$-homotopic directly from the definition. Let $Z$ and $h$ be as in the definition of the $[L]$-homotopy.

Let $\mathcal{E}=\left\{E_{G}\right\}_{G \in \mathcal{G}}$ be the collection of subsets of $Z$ defined by

$$
E_{G}=h^{-1}\left(f^{-1}(G) \cap g^{-1}(G)\right) .
$$

It is a cover of $Z$ because $f$ and $g$ are $\mathcal{G}$-close. The map $C: \mathcal{E} \rightarrow \mathcal{G}$ given by the formula $C\left(F_{G}\right)=G$ carries $\mathcal{F}$ into $\mathcal{G}$. By the definition $H=f \circ h_{\mid A} \cup g \circ h_{\mid B}$ is carried by $C$.

Since $\mathcal{G}$ is a locally finite, closed cover, so is $\mathcal{E}$. Then by [6, p. 393], since $Z$ is separable metric, there is a locally finite open cover $\mathcal{F}=\left\{F_{E}\right\}_{E \in \mathcal{E}}$ of $Z$ such that $E \subset F_{E}$ for each $E \in \mathcal{E}$ and the function $F_{E} \mapsto E$ is an invertible carrier of $\mathcal{F}$ onto $\mathcal{E}$. Let $D: \mathcal{F} \rightarrow \mathcal{E}$ be a function such that $F \supset D(F)$ for each $F \in \mathcal{F}$. Then the identity map $i d_{Z}$ is weakly carried by $D$, and $H \circ i d_{Z}$ is weakly carried by $C \circ D$. By the Weak Carrier Theorem, the map $H$ extends to the entire space $Z$ and the proof is finished.

The following is a nerve theorem for $[L]$-homotopy.

Definition. We say that topological spaces $X$ and $Y$ are $[L]$-homotopy equivalent if there exist mappings $f: X \rightarrow Y$ and $g: Y \rightarrow X$ such that $g \circ f$ and $f \circ g$ are $[L]$-homotopic to $i d_{X}$ and $i d_{Y}$ respectively. 
Theorem 3.6. If two at most $[L]$-dimensional spaces have isomorphic closed covers, regular for the class of at most $[L]$-dimensional spaces, then they are $[L]$ homotopy equivalent.

We omit the proof, which is a straightforward application of the Carrier Theorem and Lemma 3.5. similar to the proof of Theorem 3.3

Remark. Observe that an $\left[S^{n-1}\right]$-homotopy is J. H. C. Whitehead's $n$-homotopy, and by Whitehead's characterization a map between locally $(n-1)$-connected $n$-dimensional spaces is an $\left[S^{n-1}\right]$-homotopy equivalence if and only if it induces isomorphisms on homotopy groups of dimensions less than $n$. This connects Theorem 3.4 with Theorem 3.6 .

\section{REFERENCES}

[1] A. Björner. Combinatorics and topology. Notices Amer. Math. Soc., 32(3):339-345, 1985. MR0789575 (86j:05003)

[2] A. Björner. Nerves, fibers and homotopy groups. J. Combin. Theory Ser. A, 102(1):88-93, 2003. MR1970978 (2004a:55018)

[3] K. Borsuk. On the imbedding of systems of compacta in simplicial complexes. Fund. Math., 35:217-234, 1948. MR0028019 (10:391b)

[4] A. Chigogidze. Infinite dimensional topology and shape theory. In R. J. Daverman and R. B. Sher, editors, Handbook of geometric topology, pages 307-371. North-Holland, Amsterdam, 2002. MR1886673 (2003b:57030)

[5] G. de Rham. Le type simple d'homotopie d'une variété différentiable. Acta Ci. Compostelana, 4:121-126, 1967. MR0251741 (40:4968)

[6] R. Engelking. General topology, volume 6 of Sigma Series in Pure Mathematics. Heldermann Verlag, Berlin, 1989. MR.1039321 (91c:54001)

[7] R. Engelking and K. Sieklucki. Topology: a geometric approach, volume 4 of Sigma Series in Pure Mathematics. Heldermann Verlag, Berlin, 1992. MR.1199810(94d:54001)

[8] J. N. Haimov. The homotopy type of a space having a brick decomposition. Dokl. Akad. Nauk Tadzhik. SSR, 22(1):25-29, 1979. MR0539178 (80m:54011)

[9] W. Holsztyński. On spaces with regular decomposition. Bull. Acad. Polon. Sci. Sér. Sci. Math. Astronom. Phys., 12:607-611, 1964. MR0174051 (30:4258)

[10] S. Hu. Theory of retracts, Wayne State University Press, Detroit, 1965. MR0181977|(31:6202)

[11] K. Kawamura. Characterizations of Menger manifolds and Hilbert cube manifolds in terms of partitions. In H. Cook, W. T. Ingram, K. T. Kuperberg, A. Lelek, and P. Minc, editors, Continua (Cincinnati, OH, 1994), volume 170 of Lecture Notes in Pure and Appl. Math., pages 275-285. Dekker, New York, 1995. MR1326850 (96c:54026)

[12] A. T. Lundell and S. Weingram. The Topology of CW Complexes. The University Series in Higher Mathematics. Van Nostrand Reinhold Company, 1969.

[13] M. C. McCord. Homotopy type comparison of a space with complexes associated with its open covers. Proc. Amer. Math. Soc., 18:705-708, 1967. MR0216499(35:7332)

[14] A. Nagórko. Characterization and topological rigidity of Nöbeling manifolds, Ph.D. thesis, Warsaw University, 2006. Available online from http://arxiv.org/abs/math/0602574.

[15] A. Weil. Sur les théorèmes de de Rham. Comment. Math. Helv., 26:119-145, 1952. MR0050280(14:307b)

Institute of Mathematics, Polish Academy of Sciences, ul. Śniadeckich 8, 00-956 Warszawa, POland

E-mail address: amn@impan.gov.pl 